\title{
Study of Starburst/Activity/Interaction Phenomena based on the Multiple Byurakan-IRAS Galaxies
}

\author{
Gohar S. Harutyunyan and Areg M. Mickaelian
}

Byurakan Astrophysical Observatory (BAO), Armenia

Email: goharutyunyan@gmail.com, aregmick@yahoo.com

\begin{abstract}
The Byurakan-IRAS Galaxy (BIG) sample is the result of optical identifications of IRAS PSC sources at high-galactic latitudes using the First Byurakan Survey (FBS) lowdispersion spectra. Among the 1178 objects most are spiral galaxies and many have been proved to be AGN and starburst by spectroscopic observations, as well as there is a number of ULIRGs among these objects. BIG objects contain galaxy pairs, multiples, and small groups that are subject for study on the matter of the real IR-emitter in these systems. Given that these objects are powerful IR sources, they are considered as young systems indicating high rate of evolution and starburst activity exceeding $100 \mathrm{M}_{o} / \mathrm{yr}$. Spectroscopic observations show that all these systems are physical ones and we were able to measure the mutual distances and sizes for all components. Cross-correlations with the recent more accurate IR catalogues, such as 2MASS and WISE, as well as radio ones (NVSS, FIRST), provided accurate coordinates of the IR source and possibility to find the individual galaxy responsible for the IR. However, in almost half of the cases, IR position indicates the intermediate region between the components, which means that it comes from the system as a whole. Some more MW data have been matched to IR and radio to have an overall understanding on these systems. Given that these systems are mostly interacting/merging ones often containing AGN and most of them may be considered as powerful starbursts, it is possible to study starburst/activity/interaction phenomena and their interrelationship.
\end{abstract}

Keywords. IR galaxies, pairs and multiples, AGN, Starburst galaxies, interactions

Optical identifications of all IRAS PSC (IRAS 1988) sources at high galactic latitudes by means of the First Byurakan Survey (FBS, Markarian et al. 1989) have been carried out at the Byurakan Astrophysical Observatory (BAO; Mickaelian 1995). The area of the FBS with $+61^{\circ}<\delta<+90^{\circ}$ at galactic latitudes $|b|>15^{\circ}$ has been taken with a total surface of $1487 \mathrm{deg}^{2} .1577$ sources have been optically identified, 1178 sources corresponding to galaxies. The Byurakan-IRAS Galaxies (BIG) sample was constructed from these 1178 newly identified galaxies and 789 other IRAS galaxies known before in the same area, altogether 1967 objects. Among the BIG sample there are:

- AGN and starburst galaxies,

- high-luminosity IR galaxies (ULIRGs),

- compact galaxies,

- interacting pairs, multiples and groups;

- candidate "mergers",

- radio and X-ray sources, etc.

Fig. 1 gives DSS2 red images for two Byurakan-IRAS galaxies showing their multiple structure. 


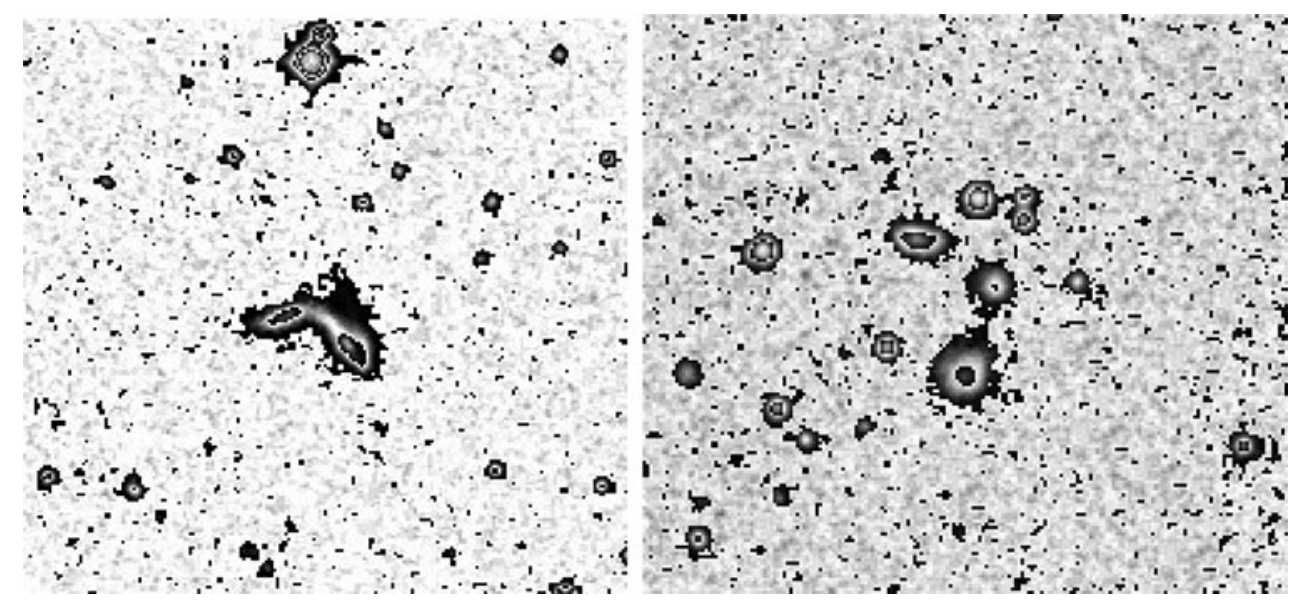

Figure 1. DSS2 red images for two Byurakan-IRAS galaxies showing their multiple structure.

We call interacting pairs, multiples and groups those having tails and bridges between the components, however only after spectroscopic observations one can consider them as real physical systems. Existence of AGN among the multiple BIG objects provides a chance for study of the galaxy evolution in sense of interrelationship between the three phenomena: starburst, interactions/merging, and nuclear activity. We have claimed in Mickaelian et al. (2001) that the maximum IR luminosity of a single spiral galaxy may not be larger than $10^{12} \mathrm{~L}_{o}$. Hence, all high-L IRAS galaxies are interacting pairs/multiples or mergers. But what is the relation of the active nucleus to the starburst (i.e. IR) activity?

Cross-correlations with the recent more accurate IR catalogues, such as 2MASS (Cutri et al. 2003) and WISE (Cutri et al. 2012), as well as radio ones (NVSS, Condon et al. 1998, FIRST, Becker et al. 2012), provided accurate coordinates of the IR sources and possibility to find the individual galaxy responsible for the IR. However, in almost half of the cases, IR position indicates the intermediate regions between the components, which means that it comes from the system as a whole. Some more MW data have been matched to IR and radio to have an overall understanding on these systems of galaxies. However, only $30 \%$ of multiple systems contain AGN, thus proving that nuclear activity in general has no direct relation to the interactions.

\section{References}

Becker R. H., Helfand D. J., White R. L., et al. 2012, ApJ 475, 479 (1997), Vizier online version 2012Feb16

Condon J. J., Cotton W. D., Greisen E. W., et al. 1998, AJ 115, 1693

Cutri, R. M., Skrutskie, M. F., van Dyk, S., et al. 2003 IPAC/CalTech

Cutri R. M., Wright E. L., Conrow T., et al. 2012, WISE All-Sky Data Release, IPAC/Caltech, VizieR On-line Data Catalog II/311

IRAS 1988, Joint IRAS Science Working Group. IRAS Catalogs, The Point Source Catalog, Version 2.0, NASA RP-1190

Markarian, B. E., Lipovetski, V. A., Stepanian, J. A., Erastova, L. K., \& Shapovalova, A. I. 1989, Com. SAO 62, 5

Mickaelian, A. M. 1995, Ap 38, 625

Mickaelian A. M., Veron-Cetty M. P. \& Veron P. 2001, Proc. IAU Symposium No. 205 (Manchester: ASP), p. 232

Veron-Cetty M. P. \& Veron P. 2010, A\& A 518, A10 